\title{
ANALYSIS OF THE TLS LONGITUDINAL FEEDBACK SYSTEM
}

\author{
W. K. Lau, C. H. Kuo, SRRC, Hsinchu, Taiwan
}

\begin{abstract}
Effectiveness of the TLS longitudinal feedback system to damp bunch phase oscillations has been studied under various operation conditions. Maximum kick voltage that is available for bunch phase correction is limited by power amplifier saturation. Operation of the feedback system in the linear and nonlinear regions of power amplifier have been simulated. In case of power amplifier saturation, the damping time is proportional to the initial oscillation amplitude. Oscillation amplitude decrease linearly before the power amplifier reaches its linear regime. The effects of hold buffer in the DAC/MUX unit and time delay of the feedback loop will also be discussed.
\end{abstract}

\section{INTRODUCTION}

The longitudinal feedback system is designed [1,2] to control beam dipole-mode longitudinal coupled-bunch instability (LCBI) in the TLS storage ring. It is believed that LCBI is driven by higher order modes of the main rf cavities. Since the growth rates of LCBI depend on cavity temperatures, tuner positions (in TLS, each cavity has an extra tuner for shifting higher order modes [3]) and beam filling patterns etc. It is not an easy task to predict exactly behavior of LCBI and put it into a realistic simulation model. In growth rate calculations, a worst case scenario is usually used. The methodology we employed to study the behavior and limitations of the feedback system operation is to treat such system ideally as independent feedback loops for each of the bunches, damping time can be found by applying an impulse excitation to the bunch. The feedback loop is considered to be effective against LCBI instability if the total damping time is shorter than the growth time of that instability. However, as mentioned above, the exact growth times of LCBI are difficult to predict. Therefore, the goal of this work is to design a digital controller which maximize damping rate. Further, the maximum available voltage to kick the beam is limited by amplifier power and kicker impedance. The feedback system is very likely to be operated in a nonlinear regime that the rf power amplifier is saturated. It is important to know how the system behaves in these circumstances. It has been discussed whether we should include a hold buffer circuitry into the DAC/MUX unit [4] to get enough loop gain. On the other hand, it is obvious that the effect of signal delay is not negligible. We reported here the works we have done in simulating the cases as described above. Section 2 describes the simulation model we are using. Section 3 summarized the results in various circumstances. Finally, the expected operational performance of the system is discussed in Section 4.

\section{SYSTEM MODEL}

Since the TLS longitudinal feedback is a bunch-by-bunch system, we can treat it as a system that consists of independent bunch phase control loops. The following figure depicted the system model we used in computer simulation.

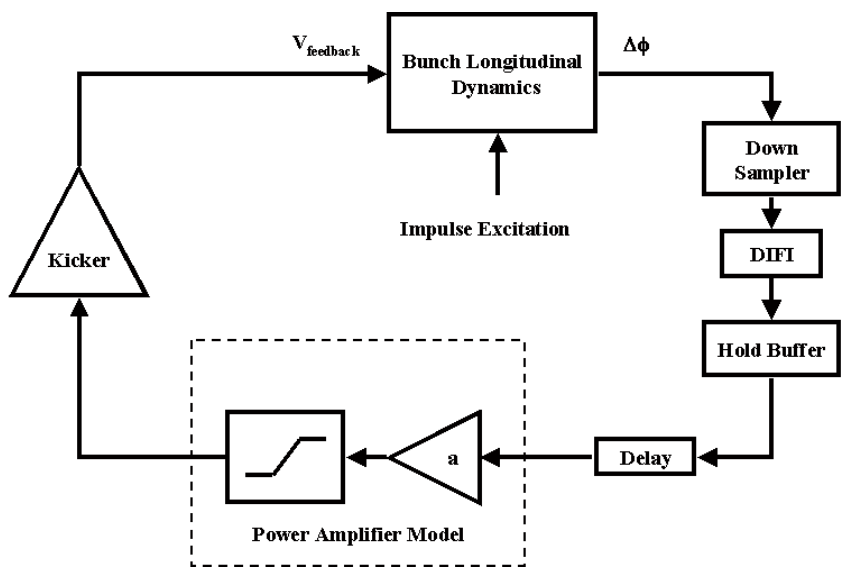

Figure 1: Longitudinal Feedback System Model used in Computer Simulation

The "bunch longitudinal dynamics" block is a tracking routine that calculates the bunch phase relative to the reference phase with feedback voltage and impulse voltage energy kick as input. In the case of TLS, input to the digital filter (DIFI) is down-sampled by 18. In this study, we tried a 5-taps FIR filter that provides a pass band around synchrotron frequency and adequate phase shift by choosing the coefficients. The filtered signal is then hold for 18 turns by the hold buffer circuit. The time needed for digital signal processing and signal synchronization require that the output from hold buffer is delayed by 18 (or its multiples) turns. However, a delay of 18 turns is used throughout the study except in the case where the effect of time delay is being studied. In order to simulate the power amplifier, the input signal is amplified by a constant gain of "a" and clipped off at a certain limit. The output power of the amplifier is converted into Efield via the longitudinal kicker. Obviously, we neglected things that may be very important such as quantization error sampled signal due to the 8-bits fast analog-todigital converter, response of kicker and mixer noise etc. Relevant parameters of the TLS longitudinal feedback system at $1.5 \mathrm{GeV}$ are recalled in Table 1 for reference. 
Table 1: Relevant Parameters of the TLS Longitudinal Feedback System

\begin{tabular}{lc} 
Beam energy & $1.5 \mathrm{GeV}$ \\
RF frequency & $500 \mathrm{MHz}$ \\
Revolution frequency & $2.5 \mathrm{MHz}$ \\
Maximum bunch number & 200 \\
Synchrotron tune & 0.0106 \\
Radiation damping time & $4.9 \mathrm{msec}$ \\
Maximum kicker voltage & $200 \mathrm{volts}$ \\
Down sampling factor & 18 \\
Number of FIR filter taps & 5 \\
\multicolumn{2}{c}{ 3 RESULTS OF COMPUTER } \\
\multicolumn{2}{c}{ SIMULATIONS }
\end{tabular}

The computer code was written in MATLAB language and was run on a WIN98 platform. The simulation results are summarized as follows:

\subsection{Beam Response without Feedback}

Without feedback, the response of a bunch is calculated at $1.5 \mathrm{GeV}$. The initial energy offset is arbitrarily chosen a small but nonzero value of $1 \mathrm{e}-6$ and the bunch phase was set at synchronous phase. An impulse kick was applied at the $2000^{\text {th }}$ turn. The response of the bunch to impulse kick obtained from the calculation in the first 18000 turns is shown in Figure 2. The damping time is $4.90 \mathrm{msec}$ or 12,250 turns. This is the natural damping time for longitudinal motion at $1.5 \mathrm{GeV}$ with the lattice that is used for user operation [5].

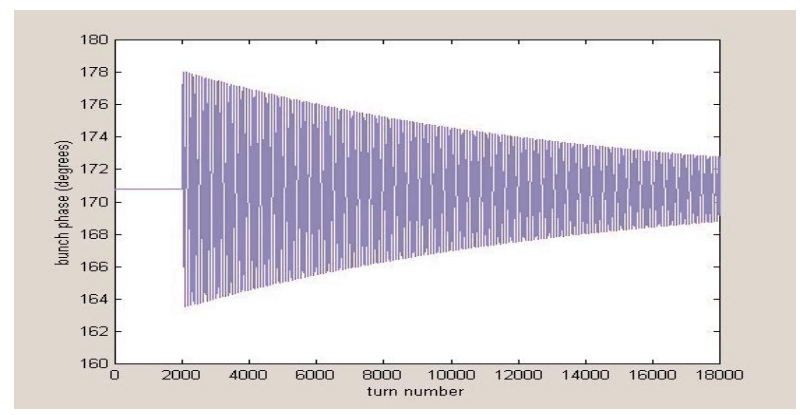

Figure 2: Damping of bunch phase oscillation due to synchrotron radiation loss

\subsection{Feedback in the Linear Regime}

By limiting the loop gain "a" within a certain value, the power amplifier is not saturated. With the same initial condition as in the case discussed above, bunch phase oscillation is damped at a faster rate than the case without feedback as expected. Figure 3 shows the response of the beam with feedback system operated in linear regime at a gain of 8. Coefficients of the FIR filter and the initial phase of the feedback voltage are optimized for fastest damping. The total damping time is $3.34 \mathrm{msec}$ or 8,357 turns.

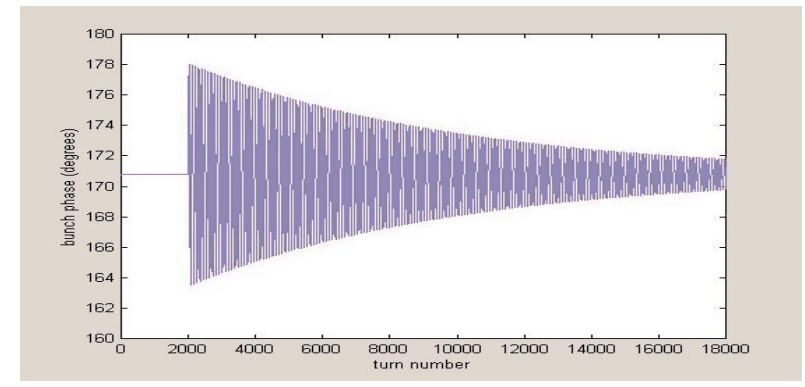

Figure 3: Damping of bunch phase oscillation with feedback system operated in the linear regime

In this specific case, the feedback loop is not strong enough for significant damping. Note also that damping time is independent of the strength of the impulse kick or initial amplitude in the linear region at a given loop gain.

\subsection{Power Amplifier Saturation}

As long as the feedback loop is kept stable, damping time can be further reduced by cranking up the loop gain. However, increase of gain is limited by power amplifier saturation. In our case, the maximum kick voltage is limited to 200 volts. Figure 4 shows an example with loop gain of 1000, the bunch phase oscillation is damped at constant rate until its amplitude is small enough that the power amplifier is operated in its linear regime. Figure 5 shows the variation of amplitude of kicker voltage in this case.

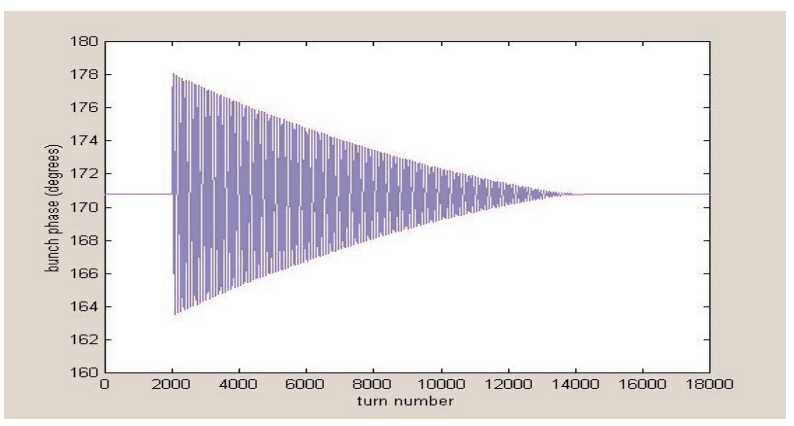

Figure 4: Damping of bunch phase oscillation in case of power amplifier saturation

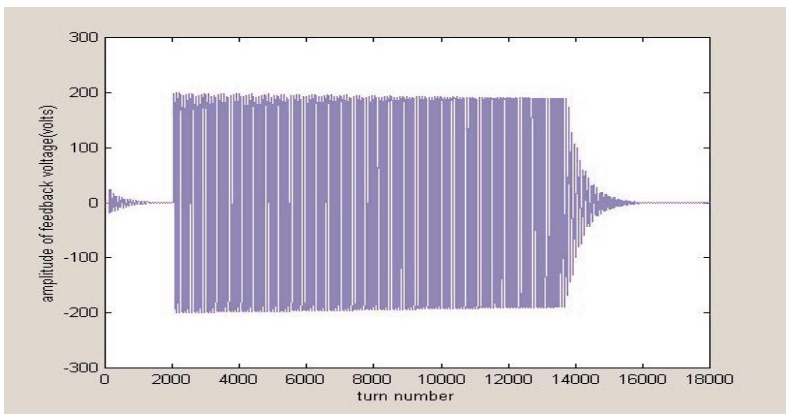

Figure 5: The corresponding amplitude of kick voltage in case of loop gain is 1000 
Damping time of the oscillation in these nonlinear operation conditions is hard to define. However, we technically defined the time required to reach an acceptable small value energy deviation. We think that our criteria should be set at 1e-4. Besides loop gain, we found also that this damping time proportional to initial amplitude of the impulse kick (Figure 6).

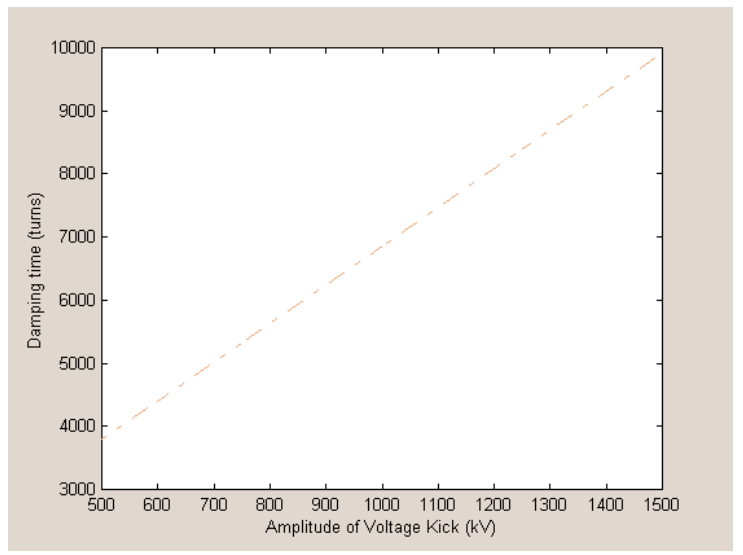

Figure 6: Damping time versus impulse kick strength at loop gain "a" equals 1000

\subsection{Effect of Hold Buffer and Time Delay}

In the simulations as discussed above, the DIFI output holds for 18 turns. However, without hold buffer, the loop gain is reduced significantly and the damping is much weaker. Therefore, a hold buffer circuitry is implemented in the DAC/MUX unit that the hold time is adjustable. A time delay of one down-sampled period (18 turns) has been set in the above simulations. Longer time delay has the advantage that more time is available for the DSP chips for calculation. The effect of more time delay (two or three periods) has also been tested. For longer delay, the phase of correction signal has been changed. One has to readjust both the phase shift of the DIFI or the initial phase of the feedback voltage to retain best performance. However, the ability of the feedback loop to damp the oscillation is weaker for a longer delay.

\section{DISCUSSIONS}

A computer code is developed to simulation the longitudinal feedback system behavior. The nonlinear characteristics of the TLS longitudinal feedback have been studied. In these simulations, quantization error resulted from analog-to-digital converter and individual device response are not included. Effects of hold buffer and time delay on system gain is also studied. We found that it is advantageous to add hold buffer circuit into DAC/MUX unit for higher loop gain. A time delay of one down sampled period is acceptable. The effect of increasing the delay time to damping rate can not be compensated by adjusting DIFI phase shift and initial phase of kick voltage. In the saturation region, the total damping time versus impulse kick strength at a given loop gain is obtained. It is linearly proportional to the kick voltage. Further studies will include effects of individual response of components, new controller design etc.

\section{REFERENCES}

[1] W. K. Lau et al., "Development of a Digital Longitudinal Damper for the TLS Storage Ring" Proceedings of Particle Accelerator Conference 1997.

[2] W. K. Lau et al., "Progress of the Longitudinal Feedback System and Associated Beam Observations in TLS", these proceedings.

[3] Ch. Wang et al., "Stabilization of the Spectral Intensity Fluctuations with the Higher Order Mode Frequency Tuners" Proceedings of Particle Accelerator Conference 1997.

[4] M. S. Yeh et al., "High Speed Digital Signal Processing Electronics for the TLS Longitudinal Feedback System" these proceedings.

[5] M. H. Wang, private discussion. 